\title{
CD117 is not always positive in infantile gastrointestinal stromal tumor
}

\author{
Jin Peng $\mathrm{He}^{1}$. Jie Xiong Feng ${ }^{1}$
}

Received: 9 May 2017 / Revised: 21 July 2017 / Accepted: 26 July 2017 / Published online: 5 March 2018

(c) Children's Hospital, Zhejiang University School of Medicine 2018

Gastrointestinal stromal tumors (GISTs) are rare in infants. GIST was presumed to originate from the interstitial cells of Cajal (ICC), which has been proven by their immunophenotype (CD117 positive). About 95\% of GISTs express CD117 and 80\% of GISTs also express CD34. They locate mostly in the stomach or the small intestine and usually cause obstruction [1]. The UK National Registry of Childhood Tumors reported the incidence of GISTs at 0.02 per million per year in children below the age of 14 years [2]. Here we will discuss a very rare case of infantile GIST with negative CD117 expression and make a review of literatures about its clinical, histopathological, and prognostic findings.

A 2 month-21-day-old female infant was transferred to our hospital because of a right quadrant abdominal mass. Previous prenatal examination revealed no abnomalities. Abdominal ultrasonography revealed a hepatonephric mass $(5.1 \times 4.7 \mathrm{~cm})$ filling the right abdominal space and presenting with internal gas signs (Fig. 1a, b). Abdominal computed tomography imaging of the infant showed a mass locating between the liver and the right kidney (Fig. 1c). Laparotomy showed an orbicular mass surrounding cecum with a size of $5 \times 4 \mathrm{~cm}$, extending from the wall of the cecum, but not intruding into the lumen. The bowel segment containing the mass was excised and an ileocecal anastomosis was performed. The pathological specimen showed a $5 \times 4 \times 4 \mathrm{~cm}$ mass surrounding the cecum. Microscopic examination of the tumor showed a spindle cell mesenchymal tumor and mild atypia (Fig. 1d, e). The mitotic rate was 1 per $50 \mathrm{HPF}$ with Ki-67 LI as $1 \%$. The tumor was completely resected with a negative margin confirmed by pathological examination post-operation. The tumor bulk was composed mostly of spindle cells staining positive for CD34, vimentin, DOG1 and partly positive for S-100. The tumor stained negative for CD117, Caldesmon, smooth muscle actin, myoglobin, $\beta$-catenin and desmin. The girl survived without recurrence for three years. To our knowledge, this is the ninth case reported a GIST in infantile period [2-9], and the second case reported an infantile GIST in the cecum (Fig. 1, Table 1).

The most common symptoms were vomiting, abdominal distention, constipation, abdominal pain and bloody stools. Multiple air fluid levels and dilated loops of bowels were presented on the abdominal X-ray examination while a mass discovered by the ultrasonography. Five male and four female infants were reported with age ranging from 1 day to 6 months. All those patients received laparotomy and bowel resection, none of them received chemotherapy or radiotherapy. One case received a reoperation because of partial necrosis of the proximal bowel end of anastomosis and consecutive leakage and two cases died of sepsis postoperation. All of the others survived free of recurrence. The GISTs located in the ileum in four cases, in the jejunum in three, and in the cecum in two. The immunohistochemistry examination showed CD117 positive in 4 cases, and CD34 positive in 5 . Three cases were classified as high risk while six cases as low risk according to the modified NIH criteria [1]. Our case is evaluated as low risk.

Complete (R0) resection of GISTs without rupturing the tumor pseudocapsule is the main aim of surgical treatment according to the reported results. Imatinib is the only treatment for GISTs that has been evaluated in the adjuvant

Jin Peng He

hejinpeng2006@126.com

1 Department of Pediatric Surgery, Tongji Hospital, Tongji Medical College, Huazhong University of Science and Technology, 1095 Jiefang Ave, Qiaokou District, Wuhan 430030, Hubei, China 

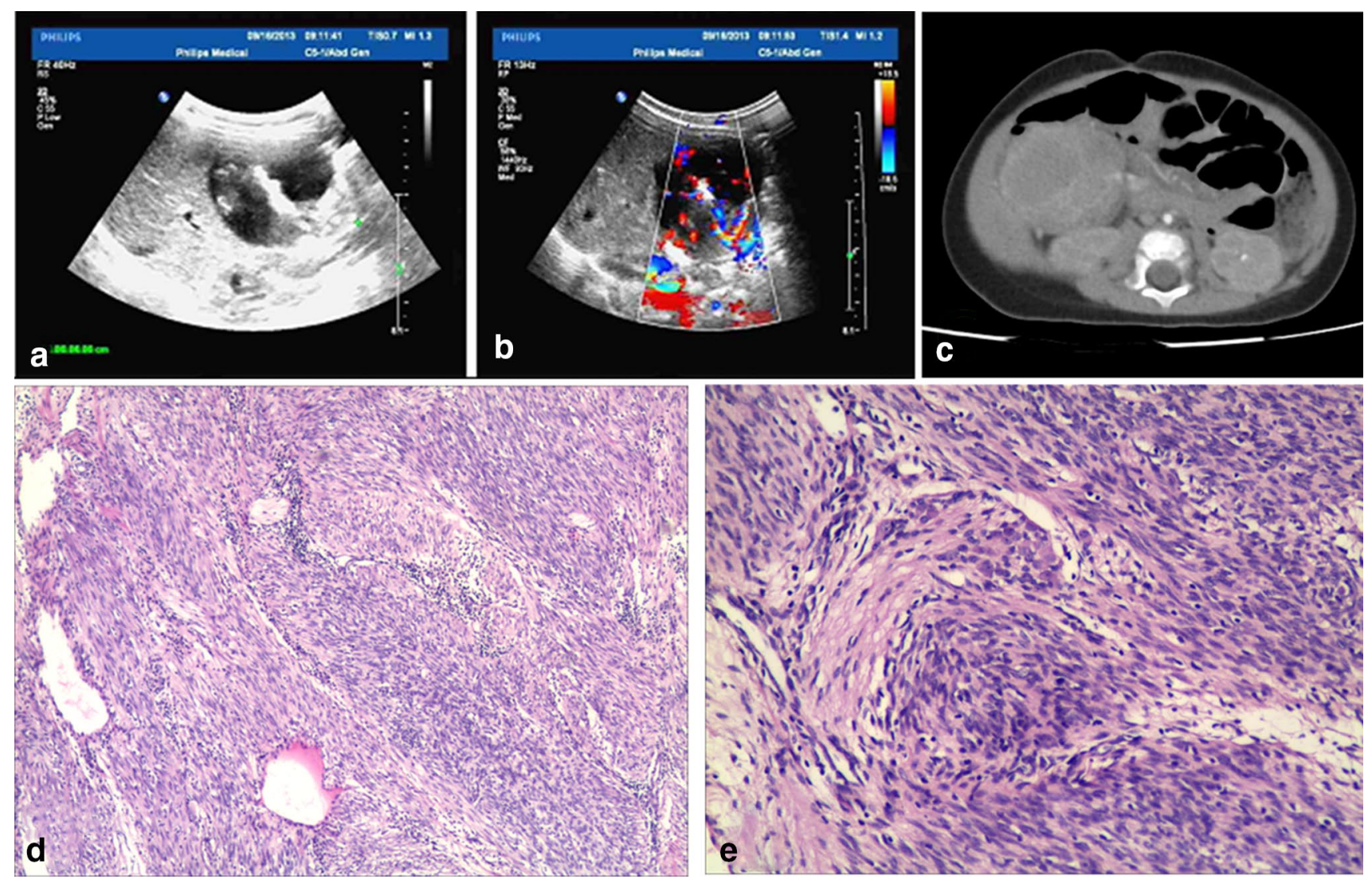

Fig. 1 The laboratory examination and H\&E staining. a \& b A heterogeneous mass on ultrasonography examination, $\mathbf{c}$ A homo-density mass on computated tomography examination, $\mathbf{d} \&$ e Spindle cells on H\&E staining (original magnification $\times 10, \times 20$ )

setting, while evidences suggesting that patients with pediatric/wild-type GIST benefit from adjuvant imatinib is unconvincing; so treatment of this group remains controversial
[10]. The treatment of GISTs in children is different from adults; furthermore, nearly half of infantile GISTs presented with CD117 negative, which is quite different with adults. 


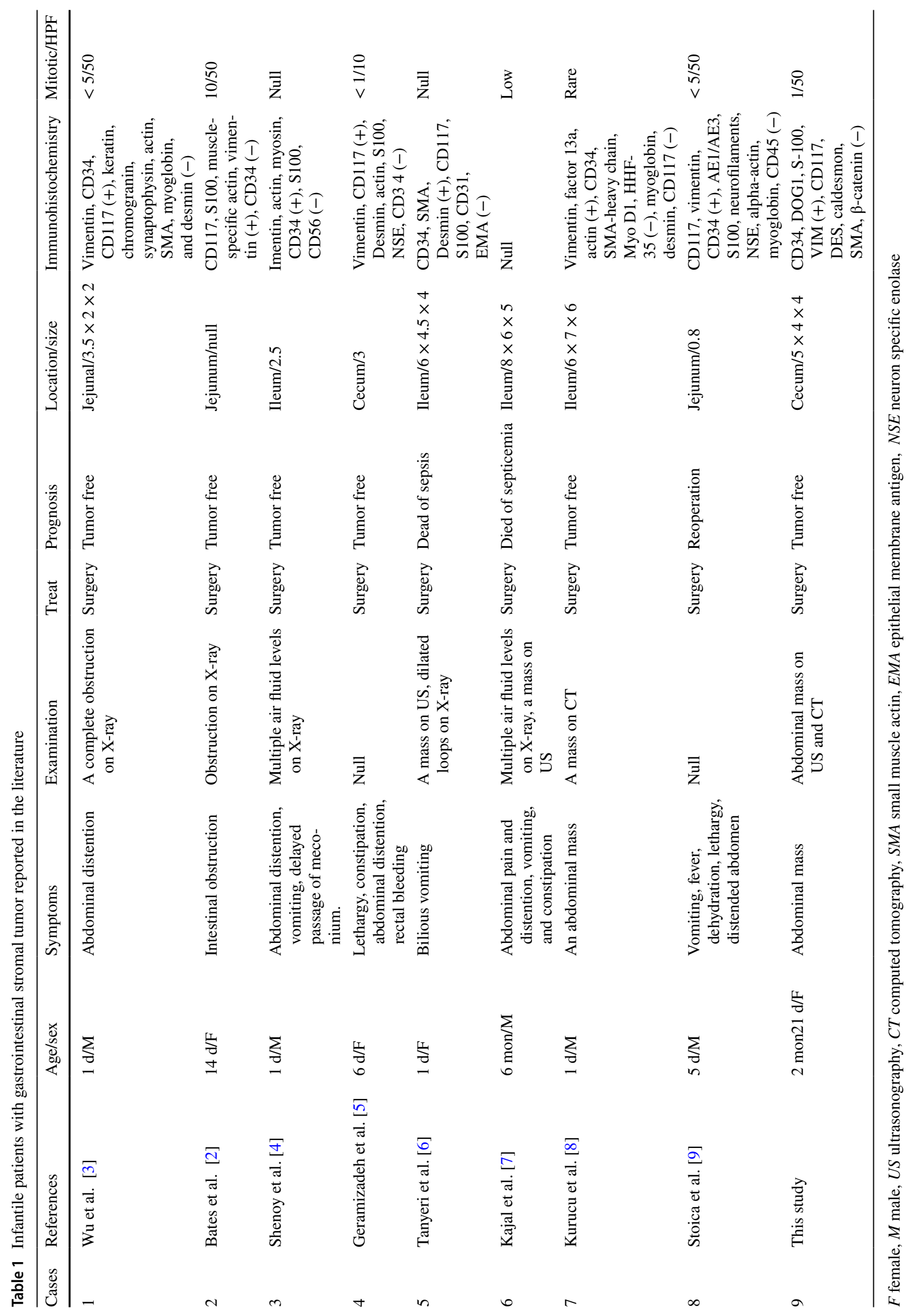


Author contributions He JP wrote the article under the supervision of Feng JX, mainly conceived and designed the study, and analyzed the data and wrote the paper. Feng JX mainly conceived and designed the study. All the authors read and approved the final manuscript.

Funding None.

\section{Compliance with ethical standards}

Ethical approval All analyses were based on previous published studies, all procedures performed in studies involving human participants were in accordance with the ethical standards of the institutional and/ or national research committee and with the 1964 Helsinki declaration and its later amendments or comparable ethical standards. For this retrospective study formal consent is not required.

Conflict of interest All the authors declare no conflict of interest.

\section{References}

1. Joensuu H, Hohenberger P, Corless CL. Gastrointestinal stromal tumour. Lancet. 2013;382:973-83.

2. Bates AW, Feakins RM, Scheimberg I. Congenital gastrointestinal stromal tumour is morphologically indistinguishable from the adult form, but does not express CD117 and carries a favourable prognosis. Histopathology. 2000;37:316-22.

3. Wu SS, Buchmiller TL, Close P, Gershman GB, Peng SK, French SW. Congenital gastrointestinal pacemaker cell tumor. Arch Pathol Lab Med. 1999;123:842-5.

4. Shenoy MU, Singh SJ, Robson K, Stewart RJ. Gastrointestinal stromal tumor: a rare cause of neonatal intestinal obstruction. Med Pediatr Oncol. 2000;34(1):70-1.

5. Geramizadeh B, Bahador A, Ganjei-Azar P, Asadi A. Neonatal gastrointestinal stromal tumor. Report of a case and review of literature. J Pediatr Surg. 2005;40:572-4.

6. Tanyeri B, Koku N, Demirci M, Karakok M, Citak EC. A rare cause of intestinal obstruction: neonatal gastrointestinal stromal tumor. Pediatr Hematol Oncol. 2011;28:155-8.

7. Rattan KN, Kajal P, Malik VS, Soni G. Multiple gastrointestinal and extragastrointestinal stromal tumors in a male infant-an extreme rarity. Trop Gastroenterol. 2012;33:285-7.

8. Kurucu N, Sari N, Celasun B, Sarihan H, Ahmetoglu A, Ilhan IE. Gastrointestinal stromal tumor in a newborn diagnosed in prenatal period: a case report and review of literature. J Pediatr Hematol Oncol. 2014;36:649-52.

9. Stoica GA, Stepan AE, Maloş A, Popa A, Georgescu CC, Stoica $\mathrm{M}$, et al. Gastrointestinal stromal tumor, jejunal atresia and stenosis in a neonate. Rom J Morphol Embryol. 2014;55:1253-7.

10. Joensuu H, Eriksson M, Sundby Hall K, Hartmann JT, Pink D, Schütte J, et al. One vs three years of adjuvant imatinib for operable gastrointestinal stromaltumor: a randomized trial. JAMA. 2012;307:1265-72. 